\title{
A rare case of dermoid cyst of the neck - clinical presentation and surgical treatment -
}

Authors:

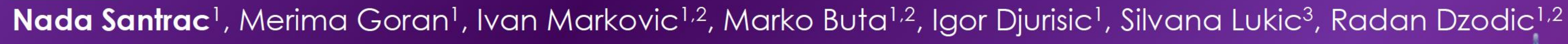

1 Institute for Oncology and Radiology of Serbia, Surgical Oncology Clinic, Pasterova 14, Belgrade, Serbia

2 University of Belgrade, School of Medicine, Dr Subotica 8, Belgrade, Serbia

3 Institute for Oncology and Radiology of Serbia, Department of Pathology, Pasterova 14, Belgrade, Serbia

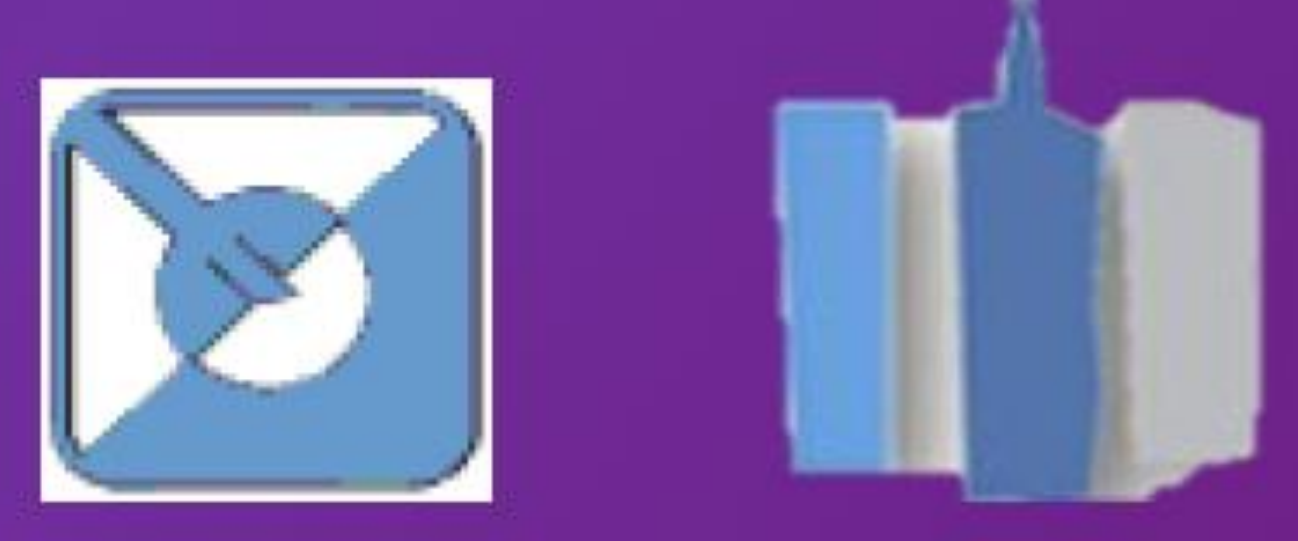

\section{INTRODUCTION}

Dermoid cysts are usually presented as solitary hamartomatous tumor, containing all skin adnexa of ectodermal origin, including hair follicles, hair, nails, sweat and sebaceous glands, or cartilage-like and bone-like structures. They can be found in the skin and subcutis of the face and neck; they can be intracranial, intraspinal, or perispinal, as well as intra-abdominal, localized in the ovary or omentum.

Cutaneous cysts most commonly appear on the head (forehead), mainly around eyes, as well as on the floor of the mouth. They can occur on the neck in the midline region, rarely.

Occasionally, the lining cyst epithelium may proliferate, with resemblance to epidermal carcinomatous proliferation, so the growth may cause misclassification of the dermoid cyst as cancer.

\section{CASE REPORT}

We present a 24-year-old female patient with a dermoid cyst in the jugulum that was operated in our Institution. Patient initially noticed painless tumor mass lower on the neck.

Physical and ultrasonical examination showed well-marginated, semi-solid, semi-cystic tumor mass in the jugulum, approximately $50 \mathrm{~mm}$ in size, localized anteriorly to infrahyoid muscles and trachea, with no connection to thyroid gland.

Patient was asymptomatic. No fine-needle aspiration biopsy was done.

Patient was operated in general anaesthesia. Through minimal skin incision on the anterior of the neck, just above the tumor mass, the cystic lesion was completely removed from the front of trachea and infrahyoid muscles, with the intact wall.

Frozen section histopathology analysis showed dermoid cyst. On definite histopathology these findings were confirmed. The cyst was $46 \times 42 \times 25 \mathrm{~mm}$ (CCXLLXAP) in size, with keratinized stratified epithelium covering the cyst wall, and sebaceous and sweat glands, as well as hair follicles and hair within.

Patient was released from hospital on the first postoperative day, with full recovery.

\section{CONCLUSION}

Dermoid cysts of the neck are rare and can often be misdiagnosed with thyroid tumors. Ultrasound and magnetic resonance imaging are helpful in making the correct differential diagnosis. Surgery is the treatment of choice, especially due to possible malignant alteration. However, surgical manipulation has to be delicate, since cyst content, especially if with bacterial infection, may spread into the surrounding tissues and cause severe complications.

Dr Nada Santrac

Institute for Oncology and Radiology of Serbia santrac.nada@gmail.com
Clinical case reports;

Thyroid/others 